\title{
Characteristics of boundary-layer transition and Reynolds-number sensitivity of three-dimensional wings of varying complexity operating in ground effect
}

\author{
Luke S Roberts ${ }^{1}$ \\ Email: 1.roberts@cranfield.ac.uk \\ Cranfield University \\ Mark V Finnis \\ Email: m.v.finnis@cranfield.ac.uk \\ Cranfield University \\ Kevin Knowles \\ Email: k.knowles@cranfield.ac.uk \\ Cranfield University
}

\begin{abstract}
The influence of Reynolds number on the aerodynamic characteristics of various wing geometries was investigated through wind-tunnel experimentation. The test models represented racing car front wings of varying complexity: from a simple singleelement wing to a highly complex 2009-specification Formula-One wing. The aim was to investigate the influence of boundarylayer transition and Reynolds-number dependency of each wing configuration. The single-element wing showed significant Reynolds-number dependency, with up to 320\% and 35\% difference in downforce and drag, respectively, for a chordwise Reynolds number difference of $0.81 \times 10^{5}$. Across the same test range the multi-element configuration of the same wing and the F1 wing displayed less than $6 \%$ difference in downforce and drag. Surface-flow visualization conducted at various Reynolds numbers and ground clearances showed that the separation bubble that forms on the suction surface of the wing changes in both size and location. As Reynolds number decreased the bubble moved upstream and increased in size, whilst
\end{abstract}

\footnotetext{
1 Aeromechanical Systems Group, Centre for Defence Engineering Cranfield University

Defence Academy of the United Kingdom

Shrivenham, SN6 8LA, UK
}

Please refer to any applicable publisher terms of use. 
reducing ground clearance caused the bubble to move upstream and decrease in size. The fundamental characteristics of boundary layer transition on the front wing of a monoposto racing car have been established.

\section{INTRODUCTION}

It is generally recognized that one of the drawbacks of many wind-tunnel tests is the inability to match full-scale Reynolds numbers, due to a sub-scale model and limited freestream air velocity. The Reynolds number represents the ratio between viscous and inertial forces, where the latter begin to dominate at higher values and the former at lower values. As viscosity acts to dampen instabilities and dissipate turbulence that naturally occurs in a fluid, testing at lower Reynolds numbers causes differences in boundary-layer state and transition, and subsequently separation points, on the test models. Such effects have been studied extensively in the aeronautical sector and shown to have significant influence on the overall airfoil performance [1-8]. Given that a wing operating in ground effect produces significantly lower pressures and stronger pressure gradients than its out-of-ground-effect counterpart, Reynolds number effects and the importance of transition could be even more important. Although some studies [9-11] have cited Reynolds number and transitional effects for ground-effect geometries, there is an overall lack of investigation into the subject.

Four configurations of three-dimensional double-element wings were examined by Jasinski \& Selig [9] through force measurements and a wake study with a 7-hole pressure probe in a fixedground facility. The work included testing the wings at a range of Reynolds numbers in addition to incidence angle and flap position. Increasing the Reynolds number between $0.7 \times 10^{6}$ and $1.3 \times 10^{6}$ caused reductions in drag and increases in downforce of $3-4 \%$.

Zerihan \& Zhang [10] tested a single-element wing at chord-based Reynolds numbers $\operatorname{Re}=2.97 \times 10^{5}$ and $\operatorname{Re}=4.47 \times 10^{5}$ at a range of ground clearances. It was found that at the higher Reynolds number more downforce was produced at low ground clearances, and drag reduced across the tested range. The 
difference between the two results was attributed to the size of the separation bubble changing with Reynolds number. Observations by Correia et al. [11], also on a single element wing but of a different airfoil profile, showed that the presence of a separation bubble altered the effective shape of the wing to aid in downforce production. Both the size and position of the bubble on the wing's surface were shown to influence downforce production, and both were dependent on Reynolds number. Additionally, Roberts et al. [12] showed, by forcing the boundary layer to transition to a turbulent state, that the aerodynamic forces and flow structures produced by the wing were significantly affected, due to the elimination of the laminar separation bubble and introduction of trailing-edge separation. This led to the postulation that fully-turbulent CFD simulations may be inappropriate for low-Reynolds-number wings in ground effect.

A computational investigation was completed by Keogh et al. [13], the aim of which was to understand compressibility effects on a simplified full open-wheel racing car geometry. The work included increasing Mach number whilst maintaining constant Reynolds number, and increasing both Mach and Reynolds numbers together; where Mach number scaling was shown to be as influential as Reynolds number scaling. The study raised an important point in that both Mach and Reynolds number effects must be considered during scaled testing. The use of fully-turbulent closure models and wall functions, however, meant that key aspects of low-Reynolds-number flows, such as the laminar boundary layer and transition, were not included in this study.

The studies discussed above have shown that transition on a wing in ground effect is separationinduced in the form of a laminar separation bubble, at least for values of Reynolds number typical of racecar wind-tunnel testing. This occurs when the laminar boundary layer can no longer overcome the adverse pressure gradient, leading to separation. Turbulence is then generated by the growth of Kelvin-Helmholtz instabilities in the separated shear layer, due to the inflectional velocity profile giving velocity shear, which causes three-dimensional motions [14-15]. Interaction with the wall of turbulent motions generated in this process prompts reattachment of the shear layer to the airfoil surface, leaving an enclosed region 
of dead air and recirculation: the bubble. PSD (Power Spectral-Density) analysis of hot-film measurements by Zhang et al. [14] showed that the reattached boundary layer is not necessarily fully turbulent, instead a transitional boundary layer can reattach and continue to develop towards fully turbulent downstream. There are instances, however, when the adverse pressure gradient is so great that it can cause what is termed a long bubble, where reattachment occurs close to the trailing edge, or a fullylaminar separation where no reattachment occurs; both significantly reduce airfoil performance. The bubble alters the pressure distribution of an airfoil because it alters the effective shape of the wing in a manner similar to an increase in camber. This causes a plateau in the pressure distribution prior to transition, followed by quick pressure recovery during the reattachment phase. A review of laminar separation bubbles by Tani [15] suggested that separation bubbles occur for chord-based Reynolds numbers in the range $\mathrm{Re}=6 \times 10^{4}$ to $\mathrm{Re}=6 \times 10^{6}$.

In our earlier studies [11-12] the effect of Reynolds-number scaling on boundary-layer transition was reported and methods of fixing transition location explored, in both cases for a single-element wing. Additionally, the importance of including transitional effects in simulations was emphasized. The current study considers such effects on increasingly complex geometries, hence covering the range of front wing geometries employed in racing car designs.

\section{DESCRIPTION OF EXPERIMENTS}

\subsection{Test Facility}

Wind tunnel experiments were conducted in a $2.7 \mathrm{~m} \mathrm{x} 1.7 \mathrm{~m}$ three-quarter open-jet, closed-return wind tunnel, located at the Defence Academy of the United Kingdom in Shrivenham, UK. The tunnel features a continuous-belt rolling road, which is synchronised with the freestream wind velocity. The freestream turbulence intensity is measured to be $0.3 \%$ at the test section center. Boundary-layer suction is applied through perforated plates ahead of the rolling road and is coupled with a knife-edge transition to the road. 
Journal of Fluids Engineering

This results in a residual boundary layer of only $1.58 \mathrm{~mm}$ thickness on the road at the leading edge of the model. An overhead strut is used to support models; this also incorporates an automated motion control system that allows ground clearance adjustments during a test. Further information on this tunnel is given by Knowles \& Finnis [16].

\subsection{Test Models}

Three model configurations, which are illustrated in Figure 1, were used for experiments in this study: a single-element wing (Figure 1a), a multi-element wing (Figure 1b), and a 2009-specification FormulaOne wing (Figure 1d). These wings represent the progression from a simple to a highly complex design, and from low to high downforce capability. The single-element wing is untwisted, untapered, with a rectangular planform and a constant GA(W)-1 airfoil cross-section. The wing has a span of $750 \mathrm{~mm}$ and chord of $119.7 \mathrm{~mm}$ and is set at an incidence of $-0.5^{\circ}$. Two rectangular endplates mounted parallel to the road are fixed to each end. The multi-element wing is of the same design as the single-element, just with the addition of two Reynard Racing "Kylie" flaps of $250 \mathrm{~mm}$ span and $160 \mathrm{~mm}$ chord orientated at $23.9^{\circ}$ incidence. The Formula-One wing is a 2009-specification 50\%-scale wind-tunnel model supplied directly by a competing constructor. The wing assembly features a main-plane of chord length $119.7 \mathrm{~mm}$, two flap elements, and a double-element auxiliary wing. Each of these wings is mounted to endplates, which feature three-dimensional curvature. Other than the image shown in Figure 1d, no further information will be provided. As all the models feature the same main-plane chord length, but different total chords, the Reynolds numbers quoted throughout this work will be based upon the main-plane chord only. This makes comparison between each configuration simpler.

All of the wings were suspended from a six-component Aerotech force balance by two vertical pylons. The force balance is fixed to the bottom of the overhead strut and housed inside a non-metric streamlined 
body to exclude it from direct interaction with the airflow (see Figure 1). Additionally, this body is representative of the nose of a monoposto racing car.

All of the geometries used are applicable to modern monoposto racing cars. The single-element wing is applicable to junior formulae cars, particularly Formula 4 as the airfoil profile is very similar, and the multi-element wing to cars used in categories such as Formula Renault 3.5 and GP2. Therefore, the geometries represent a wide range of cars across numerous series for all levels of motorsport.

\subsection{Experimental Method and Uncertainties}

Force measurements were conducted at a range of ground clearances; this is defined as the distance between the ground and the lowest point on the wing's suction surface. The pitch and roll angles of the wing were determined by measuring the distance from the ground to control points on the wing. On the single- and multi-element wings these points were pins placed into the tip where the endplate is fixed in place. For the F1 wing, for which the endplates cannot be removed, these points were the front and rear of the endplate, because this is supposed to be parallel to the ground. Based on the heights of these control points the pitch and roll of the wing was set to within a maximum error of $\pm 0.0047^{\circ}$ in pitch and $\pm 0.0038^{\circ}$ in roll for all configurations. The yaw was found by measuring between the endplate and control points at the edge of the rolling road. The accuracy of positioning the wing in yaw was $\pm 0.0023^{\circ}$.

A number of freestream air velocities were used in order to vary the Reynolds number for different tests. The control system regulated the wind speed to keep the Reynolds number constant during the test i.e. to compensate for air temperature changes. The error in the quoted Reynolds number, based on the maximum measured error during all tests, was found to be \pm 470 .

The overall uncertainty in lift and drag force coefficients at a 95\% confidence level is \pm 0.014 and \pm 0.0023 respectively for all configurations used in experiments. A parametric study of each variable, including roll angle, pitch angle, yaw angle, dynamic pressure and ground clearance, at multiple settings, 
with the variation between two settings assumed to be linear, was conducted. The total uncertainty was then calculated using the accuracy for each variable (previously stated) and results of this parametric study combined with the error of the force balance. The value quoted above is the maximum uncertainty calculated for all configurations.

Repeatability tests were conducted on each wing configuration. These consisted of ten runs of ground clearance sweeps at the same freestream velocity taken one after another. For all ground clearances the largest difference of any measurement to the mean, in terms of absolute (and percentage) lift coefficient and drag coefficient, were $0.026(0.69 \%)$ and $0.011(1.33 \%)$ respectively.

Surface-flow visualisation was conducted on the wing using a paint consisting of fluorescent pigment, oleic acid and paraffin. A spray bottle was used to apply the paint to the model immediately prior to the wind being switched on. The wing was then run in the tunnel for $20-45$ mins, depending on the test case, to allow the paraffin to evaporate and leave the flow pattern on the surface. The wing was then removed to a dark room and photographed under ultra-violet light. Nine flow visualisation tests were conducted on each wing configuration in total. These represented three ground clearances at three Reynolds numbers. The images were then transformed into an 8-bit grey-scale palette. A histogram equalisation was then applied to reduce the number of grey shades in the image. Measurements of surface-flow features were then gathered by using known dimensions, such as chord and distance between geometric features.

\section{RESULTS}

\subsection{Single-element Wing}

Downforce curves for the single-element wing are shown in Figure 2a. It is clear that the wing has significant Reynolds-number dependence. At the lowest tested Reynolds number, $1.63 \times 10^{5}$, there is no noticeable force enhancement as the wing's ground clearance reduces, instead downforce remains constant until $h / c=0.5$ where it enters the force-reduction region. This is likely to be because of the 
increase of downforce due to the constraining of flow between the wing and ground being cancelled out by the trailing-edge separation point moving upstream due to the greater adverse pressure gradient. As a result, circulation remains constant. The median Reynolds number, $2.24 \times 10^{5}$, displays what could be considered as the typical downforce curve for a single-element wing, such as that found by Zerihan \& Zhang [10] at $\operatorname{Re}=2.97 \times 10^{5}$ and $\operatorname{Re}=4.47 \times 10^{5}$. As the wing moves closer to the ground it exhibits force-enhancement and force-reduction regions. The highest Reynolds number, $2.85 \times 10^{5}$, by contrast, only exhibits force enhancement within the height range measured. Downforce continues to increase across all tested ground clearances, hence the greatest downforce is found for this Reynolds number at minimum ground clearance. Figure $2 b$ shows the corresponding drag curves. These display similar behavior in terms of ground clearance sensitivity at all Reynolds numbers in that, as ground clearance reduces, the drag coefficient increases, at an increasing rate. It should be noted, however, that at any given ground clearance a higher Reynolds number results in a lower drag coefficient; as is generally true for wings operating out of ground effect. It also appears that the drag coefficient curves are converging towards a single point as ground clearance reduces. This point, which was not measured, is presumably where the separation point, which is the cause of the downforce-reduction region, has moved upstream to the start of the adverse pressure gradient.

In both the downforce and drag curves it is clear that, at a given ground clearance, the Reynolds number dependency is reducing as the Reynolds number increases. It can be assumed, therefore, that the test Reynolds number does not have to match exactly the full-scale Reynolds number in order to give representative results.

The transition mechanism for a low-Reynolds number wing is generally a separation-induced bubble [10-11]. This feature on the single-element wing is shown by surface-flow visualisation in Figure 3. At the center-span of the wing, indicated by the red box, the flow appears highly two-dimensional and the streaklines continue in the streamwise direction. The separation and reattachment lines, which define the 
limits of the bubble, are clearly seen and remain mostly spanwise. At the wing tip, indicated by the blue box, the flow is much more three-dimensional due to the movement of air under the endplate caused by the pressure gradient across it. Owing to this, there is a spanwise component of flow contained in the bubble. Hence, whilst the center of the bubble experiences little shear stress, as indicated by the stationary paint, at the wing-tip there are horizontal streaks inside the bubble. This is because of the greater suction being generated at the central region of the wing, hence creating a pressure gradient on the surface and drawing the bubble flow inwards. This is shown by the streamlines at the separation line becoming spanwise in an inwards direction. The bubble does not extend fully to the endplate, due to the formation of the wing-tip vortex.

The size and location of the separation bubble as the Reynolds number and ground clearance vary are given in Table 1 and Table 2. The measurements were taken from the flow visualisation at the center of the wing, using the chord length and bolt hole locations as reference, but as shown in Figure 3 the bubble does not change with span until close to the tip. Table 1 includes the separation $\left(x_{s} / c\right)$ and reattachment $\left(x_{R} / c\right)$ points, and the overall length $\left(x_{L} / c\right)$ of the bubble at constant ground clearance and varying Reynolds number. These Reynolds numbers represent the lowest, median, and highest values at which force measurements were conducted. The trends are quite clear: as the Reynolds number is increased separation is delayed and the bubble length decreases. The point of separation is delayed at higher Reynolds number due to the higher energy content of the flow, meaning that the laminar boundary layer is capable of travelling further through the adverse pressure gradient before it separates. Reattachment of the boundary layer occurs sooner for the higher Reynolds number flows as turbulence production is increased due to enhanced Kelvin-Helmholtz-type instabilities being produced in the separated shear layer.

Observation of the flow-visualisation results showed that trailing-edge separation, to a varying degree, and flow reversal were noticeable on the lowest and median Reynolds numbers. However, it was not a 
Journal of Fluids Engineering

fully-laminar separation i.e. a long bubble, but in fact a second separation of the now turbulent boundary layer. For $\mathrm{Re}=2.44 \times 10^{5}$, however, flow remained attached, other than for the bubble, all the way to the trailing edge. Additionally, as the Reynolds number increases the reattachment line becomes more defined, showing there to be less unsteadiness in the reattachment phase due to the greater turbulence that is produced.

Table 1 - Separation bubble size and location at wing center-span for varying Reynolds number (single-element wing, $h / c=0.3125)$

\begin{tabular}{c|c|c|c}
$\mathrm{Re} \times 10^{-5}$ & $x_{S} / c$ & $x_{R} / c$ & $x_{L} / c$ \\
\hline 1.63 & 0.5 & 0.75 & 0.25 \\
2.04 & 0.53 & 0.78 & 0.24 \\
2.44 & 0.56 & 0.79 & 0.23
\end{tabular}

Table 2 shows the variation of bubble size and location as ground clearance is varied at constant $\operatorname{Re}=2.44 \times 10^{5}$. This high Reynolds number was chosen as it had exhibited no trailing-edge separation in previous tests, meaning that the Reynolds number effect would not be included. The key trend is that as the ground clearance is reduced the bubble moves upstream and decreases in length. The laminar separation occurs earlier at lower ground clearance due to greater adverse pressure gradient that occurs, caused by the lower pressure underneath the wing. As higher flow velocities are synonymous with the lower pressure, again, this amplifies instabilities and increases turbulence production.

Table 2 - Separation bubble size and location at wing center-span for varying ground clearance (single-element wing, $\operatorname{Re}=2.44 \times 10^{5}$ )

\begin{tabular}{c|c|c|c}
$h / c$ & $x_{S} / c$ & $x_{R} / c$ & $x_{L} / c$ \\
\hline 0.125 & 0.52 & 0.71 & 0.18 \\
0.225 & 0.55 & 0.76 & 0.21 \\
0.3125 & 0.56 & 0.79 & 0.23
\end{tabular}




\subsection{Multi-element Wing}

Downforce and drag curves for the multi-element wing at given in Figure 4. These curves appear similar in shape and trend to those found for a double-element wing (with different profiles to the one used in this study) by Zhang \& Zerihan [17]. At higher ground clearances $(h / c>0.3)$ is the force-enhancement region with concentrated tip-vortex cores [18]. Here there is negligible variation in downforce production as the Reynolds number is increased. As the ground clearance then reduces, the wing enters the forceenhancement region with burst tip-vortices [18] and some differences are seen with varying Reynolds number. It is thought that this is a result of increased vortex-induced suction as the vortices become stronger at higher Reynolds numbers. Although the wing continues to produce more and more downforce in this region at all Reynolds numbers, the rate of increase of downforce with reduction in ground clearance is less for the lower Reynolds number cases. The wing then enters the final regime, the forcereduction region, which is characterized by trailing-edge separation. As the Reynolds number is increased the ground clearance at which the wing enters the force-reduction region is generally lowered. The wing entered the force-reduction region at all tested Reynolds numbers, however, if it had been possible to test at even greater Reynolds number then it would be expected that this trend would have continued and no force-reduction would have occurred at the minimum ground clearance (as seen in Figure 2a for the single-element wing at $\operatorname{Re} \geq 2.44 \times 10^{5}$ ).

In terms of drag, the curves for which are given in Figure $4 b$, there is a Reynolds-number dependency at all tested ground clearances, albeit relatively small. The difference in drag force at any given ground clearance in the force-enhancement region remains consistent. It is only in the force-reduction region where relatively large discrepancies occur. This is, however, unsurprising given the significant difference in downforce between cases in this region.

A comparison of the laminar separation bubble size and location at the centerline of the wing for varying Reynolds numbers is given in Table 3. As the Reynolds number is increased the separation and 
reattachment points both move downstream; the bubble length, however, continues to decrease with increasing Reynolds number.

Table 3 - Laminar separation bubble size and location at wing center-span for varying Reynolds number (multielement wing, $h / c=0.3125$ )

\begin{tabular}{c|c|c|c}
$\operatorname{Re} \times 10^{-5}$ & $x_{S} / c$ & $x_{R} / c$ & $x_{L} / c$ \\
\hline 1.63 & 0.51 & 0.74 & 0.23 \\
2.04 & 0.60 & 0.76 & 0.16 \\
2.44 & 0.61 & 0.77 & 0.16
\end{tabular}

Given the lack of literature on multi-element wings with part-span flaps, it is first important to observe the fundamental flow features of such a wing. Flow visualizations of the upper and lower surfaces of the wing are shown in Figure 5. On the suction surface, which is shown in Figure 5a, the separation bubble appears again on the main plane as the transition mechanism, with laminar flow upstream and turbulent flow downstream.. Its shape, however, is much more three-dimensional in that its size and location varies across the span. It appears shortest at the center of the wing and longer near the tips. Furthermore, despite the main-plane being of constant cross-section the bubble starts further downstream in the outer region. This cannot, however, be due to the wing having the highest loading at the center. The paint inside the bubble shows a spanwise shear stress component, and this will always move towards the lowest pressure, and thus it can be seen that the lowest pressure occurs at the mid-semi span directly ahead of the flap. Despite the peak suction occurring here, the presence of the flap means that the adverse pressure gradient is not necessarily as large as in the central region because pressure-recovery occurs off the surface in the wake [19]. Laminar separation, therefore, occurs earlier in the central region due to a steeper adverse pressure gradient, despite lower peak suction values. The pressure surface of the wing also shows 
evidence of separation bubbles occurring. A bubble occurs at the rear of the main plane and close to the leading edge of the flap, as both these regions contain adverse pressure gradients.

Given the three-dimensionality of the separation bubble for the multi-element wing configuration it is not feasible to tabulate bubble size and length as in the previous section. Figure 6 shows the results of flow-visualisation tests on the wing at the lowest, median and highest Reynolds numbers tested. Trailingedge separation is apparent for the lowest Reynolds number case, however, it is limited to the central region where there are no flaps to give off-surface pressure recovery [19]. In the central region the formation of the separation bubble does show some Reynolds-number dependence, in that laminar separation occurs later and the bubble length is reduced at higher Reynolds numbers. At the outer region of the main-plane, ahead of the flap, the bubble size and position do not show any significant Reynoldsnumber dependency, as the bubble size and location appear unchanged. This was confirmed by analyzing the flow-visualisation photographs with image-processing software to find the chordwise separation location. The reattachment line is, however, once again much more defined at higher Reynolds number, indicating reduced unsteadiness.

It appears to be the presence of the flaps, which increase the downforce capability of the main-plane by altering its pressure distribution, that reduces the Reynolds-number dependency of the wing. This is deduced because it is the only difference between the single-element wing, which showed significant Reynolds-number dependency, and the multi-element wing, which shows little Reynolds-number dependency.

\subsection{Formula-One Wing}

The effect of varying Reynolds number and ground clearance on the downforce and drag coefficients of the F1-specification wing are shown in Figure 7. The downforce curve shape is similar to that of the multielement wing, with force-enhancement and force-reduction regions. The drag curve, however, is 
significantly different as drag decreases whilst the wing is still operating well within the forceenhancement region. Given that F1 wings are designed to operate in a very specific ground clearance range it is likely that this drag reduction is in that region. Due to the complex design of the wing the trends in Figure 7 could be due to a number of features; identifying such features is not part of the scope of this work and hence will not be explored further.

Figure 8 shows flow visualisation of the F1 wing's lower surfaces at the lowest and highest Reynolds numbers and constant ground clearance $h / c=0.3125$. The differences between the two cases are mostly negligible, although this is expected given that the forces they produce are so similar. Given that the separation line, which defines the start of the separation bubble, is unchanged on the main plane between the two, it is likely that this represents the start of the adverse pressure gradient. If the bubble is indeed starting at the same point at varying Reynolds number then this will be some contributor in erasing Reynolds-number dependency. Looking at the central region of the wing, the bubble for $\operatorname{Re}=2.44 \times 10^{5}$, is slightly shorter, as would be expected, but clearly this is not having a large effect on the overall force production. This could be because the location of the bubble is more important than its length; this would make sense since occurring closer to the peak suction means that the suction value it maintains is greater. The other possibility is that the bubble height is very small and thus the cambering effect that it provides to the wing is insignificant in terms of overall force production. The one drawback of surface-flow visualisation is that it gives no information on what is happening a very small distance from the surface; this can lead to features that appear significant being energetically quite small. This does not mean that the flow visualisation results are insignificant, just that further analysis is required to understand the bubble's effect on the flow-field.

Flow visualisation of the wing at varying ground clearances and constant Reynolds number is shown in Figure 9. The leading edge of the bubble becomes much straighter, in the spanwise direction, for the lower ground clearance; this can be assumed to be due to laminar separation occurring immediately upon 
the start of the adverse pressure gradient. Although the size of the bubble does not appear to change much in the central region, it is clearly shorter in the outer region for the lower ground clearance. No trailingedge separation occurs, however. The bubble that appears on the flap has been eliminated at the lower ground clearance. Given that the presence of a bubble has been shown to increase effective camber of the wing and aid in downforce production, this could be a contributor to the downforce loss. The main loss, however, appears to be the bursting of tip vortices. In Figure $9 \mathrm{~b}$ the tip vortex leaves an imprint on the endplate, which shows a stable rotational flow whilst at the lower ground clearance in Figure 9a the flow appears much more chaotic, indicating vortex bursting. Additionally, the footplate channel (labelled in Figure 9), which is designed to house a vortex, shows signs of separated flow at the lower ground clearance. This indicates that flow moving around the endplate is not rolling into the tight, concentrated vortex it was at higher ground clearances.

\section{DISCUSSION}

The results presented in the previous sections appear to show high Reynolds-number dependency in the force results of the single-element wing, but virtually none for the F1 wing. Figure 10 shows the differences in downforce and drag between tests conducted at $\operatorname{Re}=1.63 \times 10^{5}$ and $\operatorname{Re}=2.44 \times 10^{5}$. In terms of downforce, it can be seen that there is up to $320 \%$ difference in the downforce coefficients between the two Reynolds numbers. Whilst this maximum does occur at the lowest ground clearance, which is in the force-reduction region, the minimum difference is still $15 \%$, and this increases almost exponentially as ground clearance reduces. By contrast, the multi-element and F1 wings each have a difference in downforce coefficient that is less than $2 \%$ across the majority of the ground clearance test range, with a maximum difference of less than $6 \%$ occurring at the minimum ground clearance. The Reynolds-number dependency of the drag coefficient shows a similar tendency, in that for the single- 
element wing the maximum and minimum differences between the two cases are $35 \%$ and $28 \%$ respectively; whilst for the multi-element and F1 wings the difference is always less than $3 \%$.

It is clear that there is a large difference in the Reynolds-number dependency of the single- and multielement wings despite them being quite similar geometrically; the only difference being the inclusion of the flap elements. The difference between the multi-element and Formula-One wings, however, is much greater in terms of geometric complexity. Prior to the force-reduction region, which occurs at approximately $h / c=0.225$ for both wings, the Reynolds-number dependency for the F1 wing is smaller, for both downforce and drag, than that for the multi-element wing. Considering only the forceenhancement region $(h / c>0.225)$, the $\mathrm{F} 1$ wing never experienced a difference in downforce greater than $1 \%$ and drag greater than $1.75 \%$. In the same range, however, the multi-element wing experienced differences of $2-4 \%$ in downforce and up to $3 \%$ in drag.

Diwan \& Ramesh [20] showed that as Reynolds number increases, a laminar separation bubble's length, height and aspect ratio all decrease. Flow visualisation results here have shown the bubble length to reduce with increasing Reynolds number, although experimental off-surface measurements of bubble height could not be made. It is assumed, however, that Diwan \& Ramesh's results hold true in this case and that it could be stated that the smaller bubble that occurs at higher Reynolds number means that its effect on the wing, which is to alter its effective shape to that of a wing with more camber, reduces. If a Reynolds number based on the local flow velocity under the wing, rather than that of the freestream air were considered, the multi-element wing would be operating at a much higher Reynolds number - this is evidenced by the much higher suction it must be generating in order to produce the higher downforce levels. Comparing Table $1 \&$ Table 3 it can be seen that for the multi-element wing the bubble length is much shorter. Therefore, based on the argument that the bubble aspect ratio decreases at higher local Reynolds number, it is proposed that the bubble's influence on the effective shape of the wing is significantly reduced for the multi-element wing. This would lead to the notion that the Reynolds-number 
dependency of the wing is linked to the level of downforce it produced; in which the more downforce is produced, the less significant scale effects are. This is supported by the observation that the F1 wing produced the highest downforce of the wings tested and had the lowest Reynolds-number dependency. Although it cannot be conclusively shown in the present study, due to the lack of off-surface measurements, it is postulated that the increased upwash component in the flow from the higherdownforce-producing wings also aids in suppression of the bubble's influence in addition to the higher flow velocity.

\section{CONCLUSIONS}

Although previous studies have indicated that boundary-layer transition occurs on the suction surface of a wing operating at low Reynolds number and in ground effect through a laminar separation bubble, and Reynolds-number scaling effects have been touched upon previously, there has been a lack of explicit study into such phenomena. In the present study, three three-dimensional wing geometries representative of monoposto racing car front wings of varying complexity were tested at varying Reynolds numbers and ground clearances.

It was found that the more complex the wing, and equally the more downforce the wing produced, the less Reynolds-number dependency was observed. The single-element wing showed considerable Reynolds-number dependency, both in the force-enhancement and force-reduction regions, whilst for the multi-element and F1-specification wings differences were only particularly present in the force-reduction region. The wings all featured laminar separation bubbles, the size and location of which were shown to be dependent on both Reynolds number and ground clearance, the latter being akin to a change in pressure gradient magnitude. As the Reynolds number reduced, the bubble moved upstream and increased in size, as the lower energy in the flow caused the laminar boundary layer to separate earlier and the greater viscous effects reducing the production of turbulence. As ground clearance reduced the bubble moved 
upstream and became shorter in length. This was attributed to the larger adverse pressure gradient causing earlier laminar separation, but with the higher flow velocity under the wing leading to an increase in Kelvin-Helmholtz instabilities to cause earlier turbulent reattachment.

Significant Reynolds-number dependency was exhibited by the single-element wing; at the lowest tested Reynolds number the wing never displayed force-enhancement phenomena. It could be argued that this Reynolds number was too low for the wing to operate properly. The fact that the multi-element and F1 wings worked at this Reynolds number in a manner negligibly different from the highest tested Reynolds number shows that it can be appropriate to test at reduced Reynolds numbers in wind tunnels.

The multi-element wing produced significantly more suction and showed a reduction in the length of the separation bubble. Based on previous studies it is proposed that the higher flow velocity under the wing also leads to a reduction in the bubble height, which in turn leads to a reduced influence on the wing. The off-surface flow fields of the multi-element and F1 wings are considerably more complex than that of the single-element wing, due to the multiple vortices that are generated. It is possible that these vortices may also be a contributing factor to the Reynolds-number dependency of the wing configuration, given that vortex-induced suction is also a force-enhancement mechanism for the wing. Therefore, as Reynolds number increases, force-enhancement due to the bubble altering the wing's effective shape is reduced, and force-enhancement due to vortex-induced suction increases. It must be concluded then, that there is a clear relationship between the downforce capability of the wing configuration and its Reynolds-number sensitivity, as both these factors are Reynolds-number dependent. The overlying trend through this work, is that as the downforce level was increased, which is somewhat linked to the complexity of the geometry, the Reynolds-number sensitivity of the wing configuration diminished.

The characteristics of boundary layer transition on wings operating in ground effect have been established. Additionally, it has been shown that different wing geometries possess significantly different sensitivities to Reynolds number. 


\section{ACKNOWLEDGMENT}

The first author acknowledges the support of a Cranfield Defence and Security research bursary.

\section{NOMENCLATURE}

$\begin{array}{ll}A & \text { Wing planform area } / \mathrm{m}^{2} \\ c & \text { Wing main-plane chord } / \mathrm{m} \\ C_{L} & \text { Lift coefficient }\left(\frac{L}{q_{\infty} A}\right) \\ C_{D} & \text { Drag coefficient }\left(\frac{D}{q_{\infty} A}\right) \\ D & \text { Drag force } / \mathrm{N} \\ h & \text { Ground clearance } / \mathrm{m} \\ L & \text { Lift force (positive upwards) } / \mathrm{N} \\ M & \text { Mach number } \\ q_{\infty} & \text { Freestream dynamic pressure } / \mathrm{Nm}^{-2} \\ R e & \text { Chord-based Reynolds number }\left(\frac{U_{\infty} c}{v}\right) \\ U_{\infty} & \text { Freestream velocity } / \mathrm{ms}^{-1} \\ x_{S} / c & \text { Normalized chordwise separation point } \\ x_{R} / c & \text { Normalized chordwise reattachment point } \\ x_{L} / c & \text { Normalized chordwise bubble length }\end{array}$

Cartesian coordinates ( $\mathrm{x}$ - downstream, $\mathrm{y}$ - vertically down, $\mathrm{z}$ - horizontal).

$x, y, z \quad$ Origin at leading edge and centerline of the wing.

$\Delta C_{D} \quad$ Percentage change in $C_{D}$ between Re values $\left(\frac{C_{D_{R e=1.63 \times 10^{5}}}-C_{D_{R e=2.44 \times 10^{5}}}}{C_{D_{R e=1.63 \times 10^{5}}}}\right)$

$\Delta-C_{L} \quad$ Percentage change in $-C_{L}$ between Re values $\left(\frac{\left(-C_{L_{R e=1.63 \times 10^{5}}}\right)-\left(-C_{L_{R e=2.44 \times 10^{5}}}\right)}{\left(-C_{L_{R e=1.63 \times 10^{5}}}\right)}\right)$

$v \quad$ Kinematic viscosity $/ \mathrm{m}^{2} \mathrm{~s}^{-1}$

$\rho \quad$ Density $/ \mathrm{kg} \mathrm{m}^{-3}$ 
[1] Hu, H., and Yang, Z., 2008, "An Experimental Study of the Laminar Flow Separation on a LowReynolds-Number Airfoil.” ASME J. Fluids Eng, 130(5): 051101.

[2] O'Meara, M. M., and Mueller, T. J., 1987, "Laminar Separation Bubble Characteristics on an Airfoil at Low Reynolds Numbers.” AIAA Journal, 25(8), pp. 1033-1041.

[3] Burgmann, S., Brucker, C., and Schroder, W., 2006, "Scanning PIV Measurements of a Laminar Separation Bubble." Experiments in Fluids, 41(2), pp. 319-326.

[4] Karasu, I., Genç, M. S., and Açikel, H. H., 2013, "Numerical Study on Low Reynolds Number Flows Over an Aerofoil.” J Appl Mech Eng., 2(5).

[5] Lee, D., Nonomura, T., Oyama, A., and Fujii, K., 2015, "Comparison of Numerical Methods Evaluating Airfoil Aerodynamic Characteristics at Low Reynolds Number." AIAA Journal of Aircraft, 52(1), pp. 296-306.

[6] Mitra, A., and Ramesh, O. N., 2013, "The Role of Laminar Separation Bubbles on Low Reynolds Number Airfoils.” AIAA Paper 2013-0059, 51st AIAA Aerospace Sciences Meeting Including the New Horizons Forum and Aerospace Exposition. Grapevine, TX, USA.

[7] Cadieux, F., Domaradzki, J. A., Sayadi, T., and Bose, A., 2014, "Direct Numerical Simulations and Large Eddy Simulation of Laminar Separation Bubbles at Moderate Reynolds Numbers.” ASME J. Fluids Eng, 136(6): 060902.

[8] Poels, A., Rudmin, D., Benaissa, A., and Poirel, D., 2015, "Localisation of Flow Separation and Transition over a pitching NACA0012 Airfoil at Transitional Reynolds Numbers Using Hot-Films." ASME J. Fluids Eng (Accepted Manuscript). DOI:10.1115/1.4031008

[9] Jasinski, W. J., and Selig, M. S., 1998, "Experimental Study of Open-Wheel Race Car Front Wings," SAE Paper 98-3042.

[10] Zerihan, J., and Zhang, X., 2000, "Aerodynamics of a Single-Element Wing in Ground Effect," AIAA Journal of Aircraft, 37(6), pp. 1058-1064.

[11] Correia, J., Roberts, L. S., Finnis, M. V., and Knowles, K., 2014, "Scale Effects on a Single-Element Inverted Wing in Ground Effect,” The Aeronautical Journal, 118(1205), pp. 797-809.

[12] Roberts, L. S., Correia, J., Finnis, M. V., and Knowles, K., 2014, "Investigation of Forcing Boundary Layer Transition on a Single-Element Inverted Wing in Ground Effect," International Vehicle Aerodynamics Conference, Paper C1385 015. Loughborough, UK.

[13] Keogh, J., Doig, G., and Diasinos, S., 2014, "Flow Compressibility Effects Around an Open-Wheel Racing Car,” The Aeronautical Journal, 118(1210), pp. 1409-1430.

[14] Zhang, X. F., Mahallati, A., and Sjolander, S. A., 2002, "Hot Film Measurements of Boundary Layer Transition, Separation and Reattachment on a Low-Pressure Turbine Airfoil at Low Reynolds Numbers," AIAA Paper 2002-3643, 38th AIAA/ASME/SAE/ASEE Joint Propulsion Conference \& Exhibit, Indianapolis, Indiana. 
[15] Tani, I., 1974, "Low-speed Flows involving Bubble Separations," Progress in Aerospace Sciences 5, pp. 70-103.

[16] Knowles, K., and Finnis, M. V., 1998, "Development of a New Open-jet Wind Tunnel and Rolling Road Facility," 2nd MIRA International Conference on Vehicle Aerodynamics, Coventry, UK. MIRA Ltd., Nuneaton.

[17] Zhang, X., and Zerihan, J., 2003, "Aerodynamics of a Double Element Wing in Ground Effect," AIAA Journal, 45(6), pp. 1007-1016.

[18] Zhang, X., and Zerihan, J., 2004, "Edge Vortices of a Double-Element Wing in Ground Effect," AIAA Journal of Aircraft, 41(15), pp. 1127-1137.

[19] Smith, A. M. O., 1975, “High-Lift Aerodynamics,” Journal of Aircraft, 12(6), pp 501-530.

[20] Diwan, S. S., and Ramesh, O. N., 2007, "Laminar Separation Bubbles: Dynamics and Control," Sadhana, 32(1-2), pp 103-109. 
Figure 1 - Diagram of a) single-element wing, b) multi-element wing, c) cross-section view of singleand double-element aerofoils, and d) Formula One wing....

Figure 2 - Single-element wing: variation with ground clearance $(h / c)$ of a) downforce and b) drag at

varying Reynolds number

Figure 3 - Suction-surface flow visualisation of single-element wing. Flow moving from top to bottom,

wing tip on the right $\left(h / c=0.3125, \operatorname{Re}=2.03 \times 10^{5}\right)$

Figure 4 - Multi-element wing: variation with ground clearance $(h / c)$ of a) downforce and b) drag at

varying Reynolds number

Figure 5 - Flow visualisation of a) suction surfaces and b) pressure surfaces of multi-element wing $(h / c$

$\left.=0.3125, \mathrm{Re}=2.44 \times 10^{5}\right)$......

Figure 6 - Suction-surface flow visualisation of multi-element wing at $h / c=0.3125$ for varying

Reynolds numbers a) $\operatorname{Re}=1.63 \times 10^{5}$, b) $\operatorname{Re}=2.04 \times 10^{5}$, and c) $\operatorname{Re}=2.44 \times 10^{5}$. .25

Figure 7 - Formula-One wing: variation with ground clearance $(h / c)$ of a) downforce and b) drag at

varying Reynolds number

Figure 8 - Suction-surface flow visualisation of Formula-One wing at $h / c=0.3125$ for varying

Reynolds numbers a) $\mathrm{Re}=1.63 \times 10^{5}$ and b) $\mathrm{Re}=2.44 \times 10^{5}$ .26

Figure 9 - Flow visualisation of F1 wing suction-surfaces (top) and inner endplate (bottom) at a) $h / c=$

0.125 and $\mathrm{b}) h / c=0.3125\left(\mathrm{Re}=2.44 \times 10^{5}\right)$

Figure 10 - Reynolds-number dependency of downforce and drag coefficients of a) single-element wing and b) multi-element and F1 wing configurations (experimental, percentage difference between $\mathrm{Re}=$ $2.44 \times 10^{5}$ and $1.63 \times 10^{5}$ results)

Table 1 - Separation bubble size and location at wing centre-span for varying Reynolds number (singleelement wing, $h / c=0.3125$ )

Table 2 - Separation bubble size and location at wing centre-span for varying ground clearance (singleelement wing, $\mathrm{Re}=2.44 \times 10^{5}$ )

Table 3 - Laminar separation bubble size and location at wing centre-span for varying Reynolds number (multi-element wing, $h / c=0.3125$ ) 


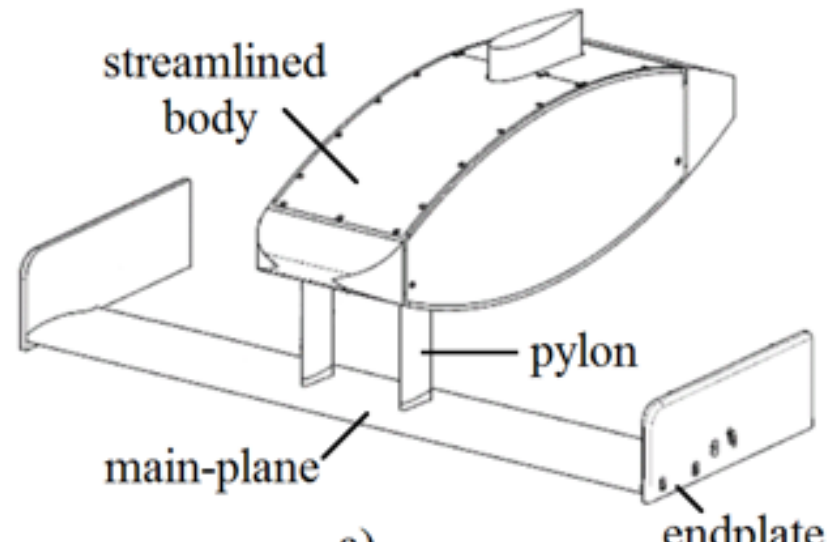

a)

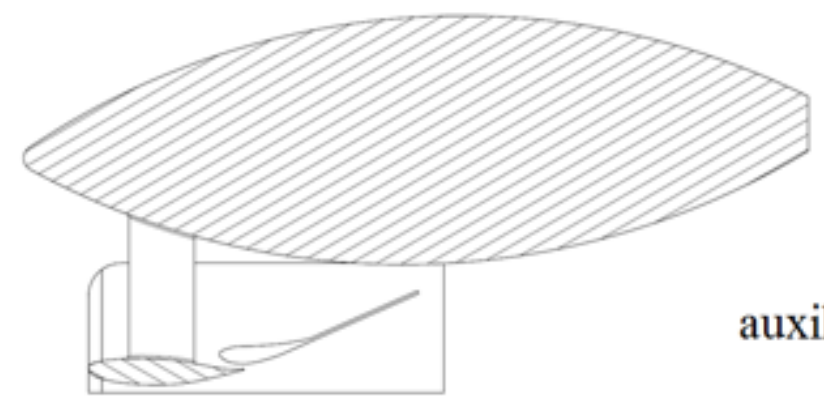

c)

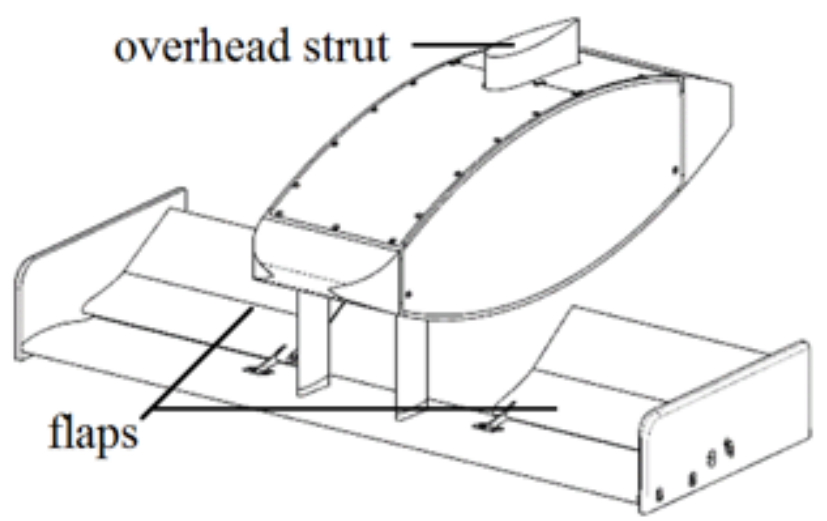

b)

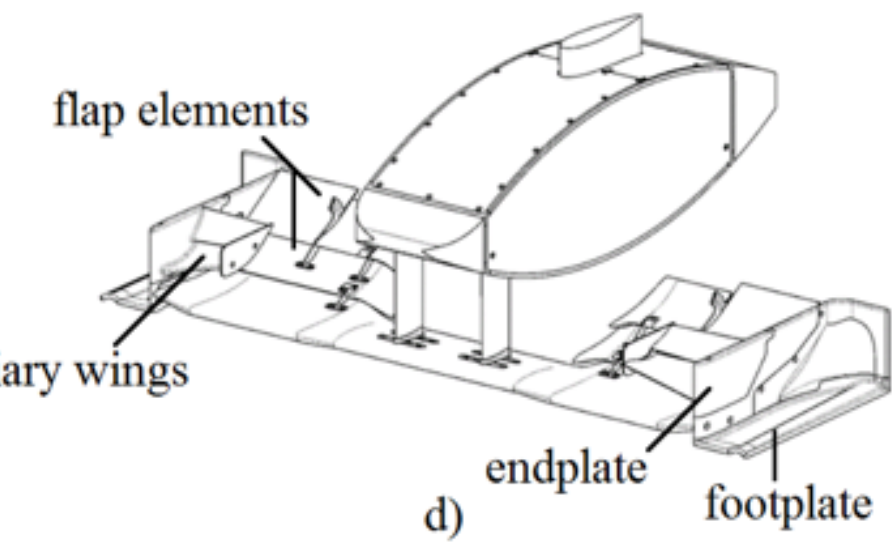

Figure 1 - Diagram of a) single-element wing, b) multi-element wing, c) cross-section view of single- and double-element airfoils, and d) Formula-One wing

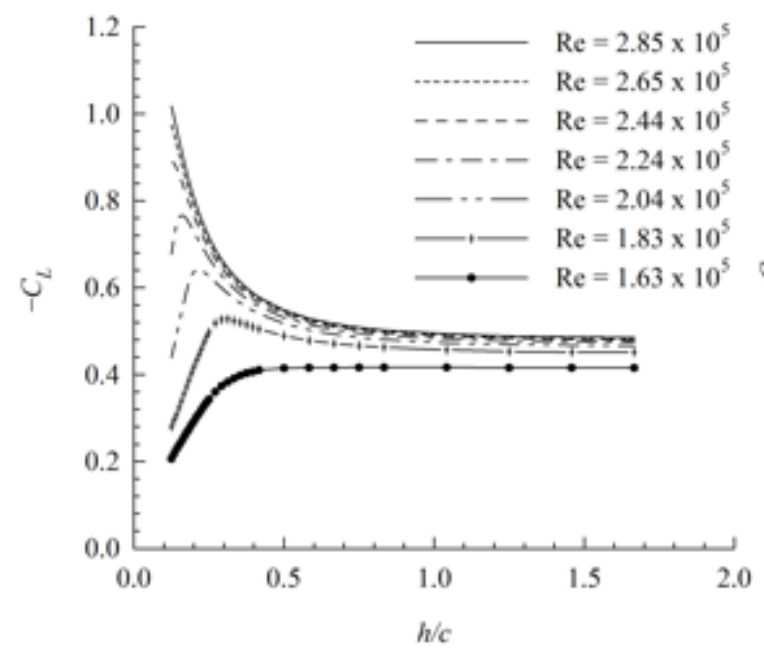

a)

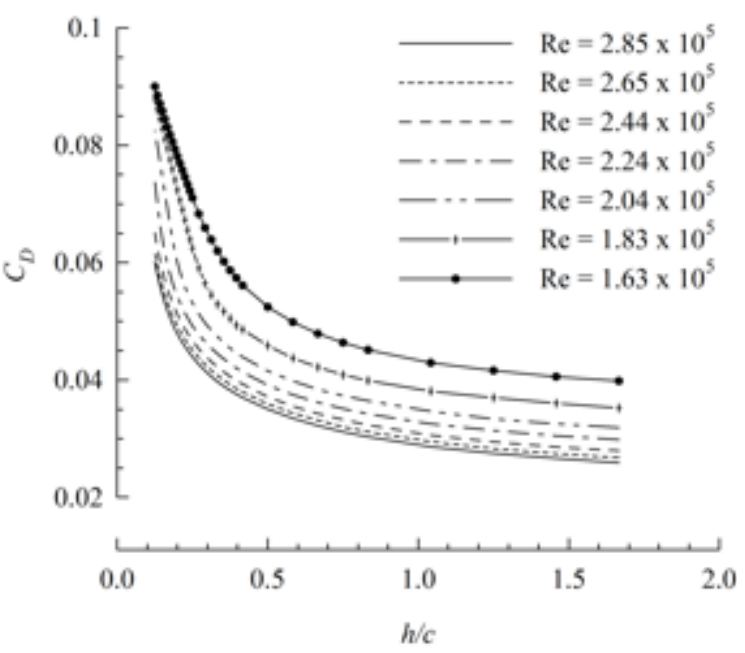

b) 
Journal of Fluids Engineering

Figure 2 - Single-element wing: variation with ground clearance $(h / c)$ of a) downforce and b) drag at varying Reynolds number

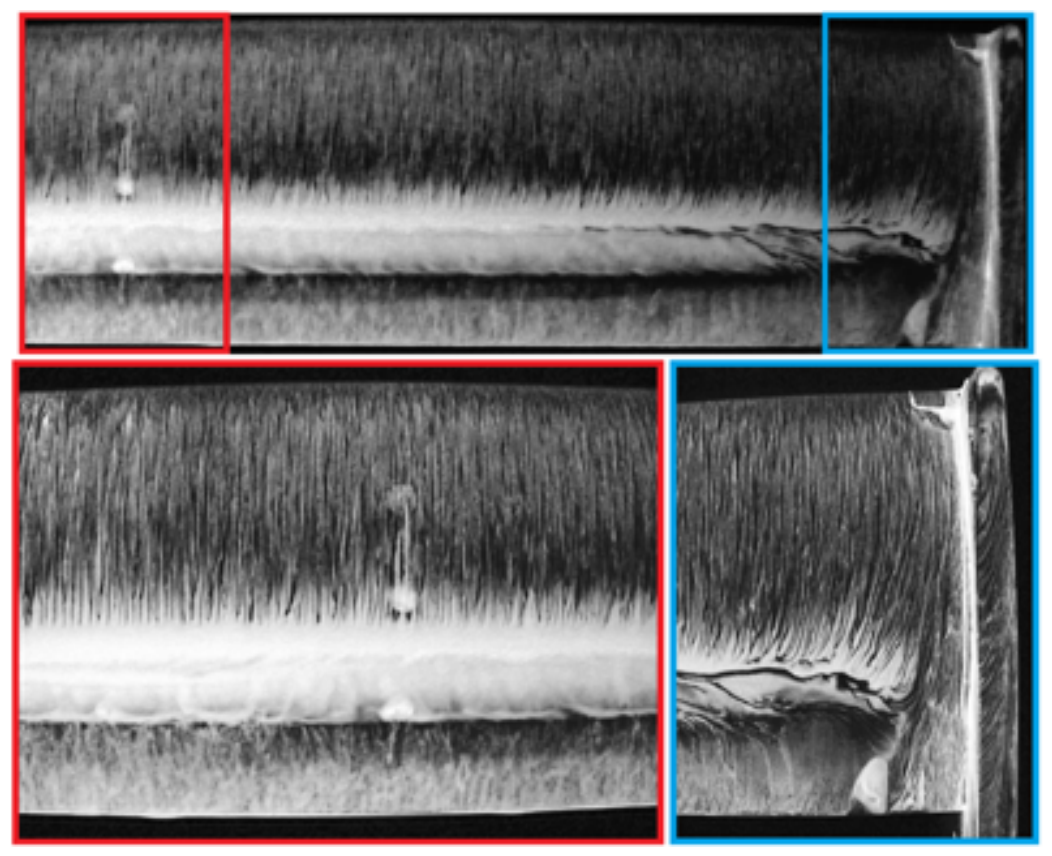

Figure 3 - Suction-surface flow visualisation of single-element wing. Flow moving from top to bottom, wing tip on the $\operatorname{right}\left(h / c=0.3125, \operatorname{Re}=2.03 \times 10^{5}\right)$

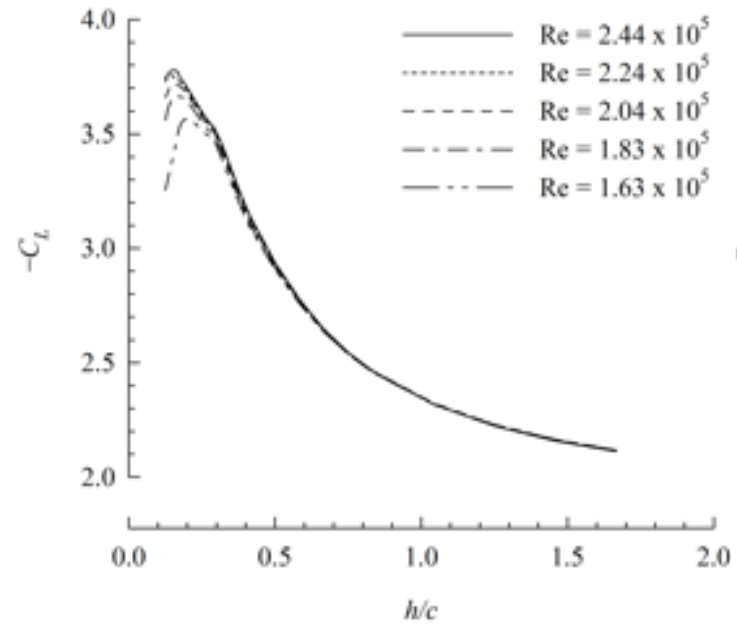

a)

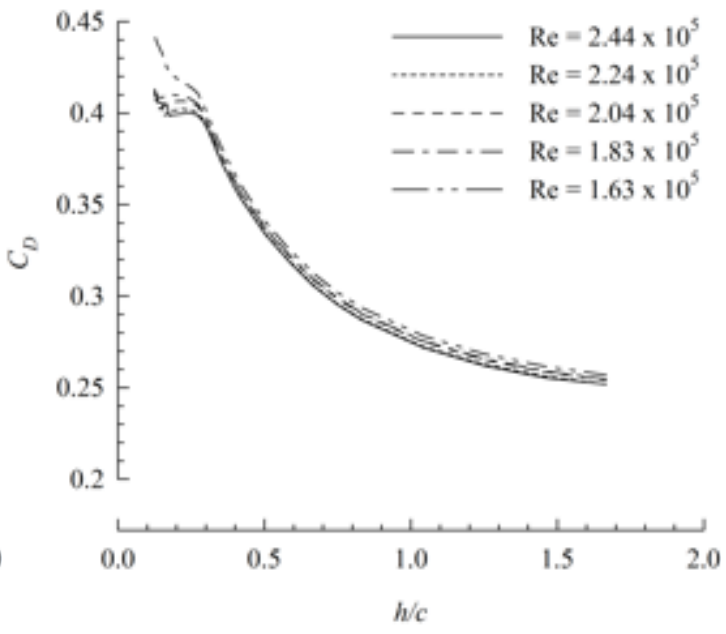

b)

Figure 4 - Multi-element wing: variation with ground clearance $(h / c)$ of a) downforce and b) drag at varying Reynolds number 


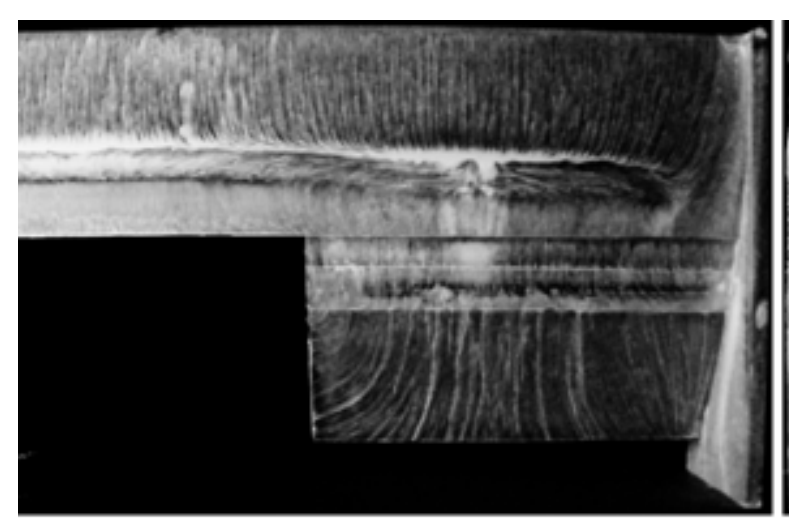

a)

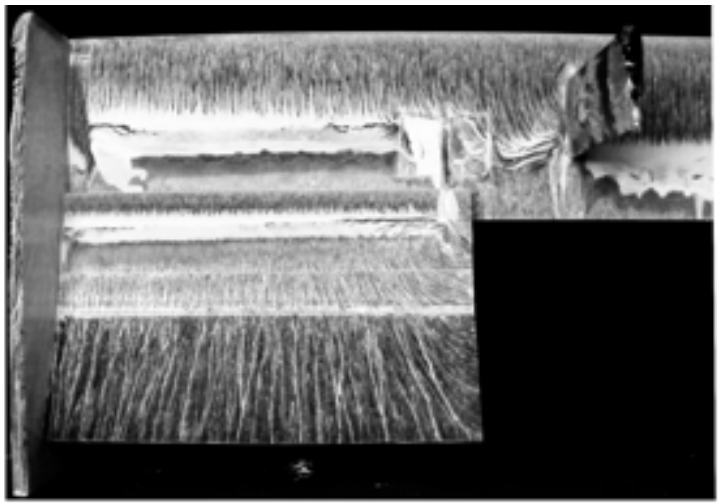

b)

Figure 5 - Flow visualisation of a) suction surfaces and b) pressure surfaces of multi-element wing $(\mathrm{h} / \mathrm{c}=\mathbf{0 . 3 1 2 5}, \mathrm{Re}=$ $\left.2.44 \times 10^{5}\right)$

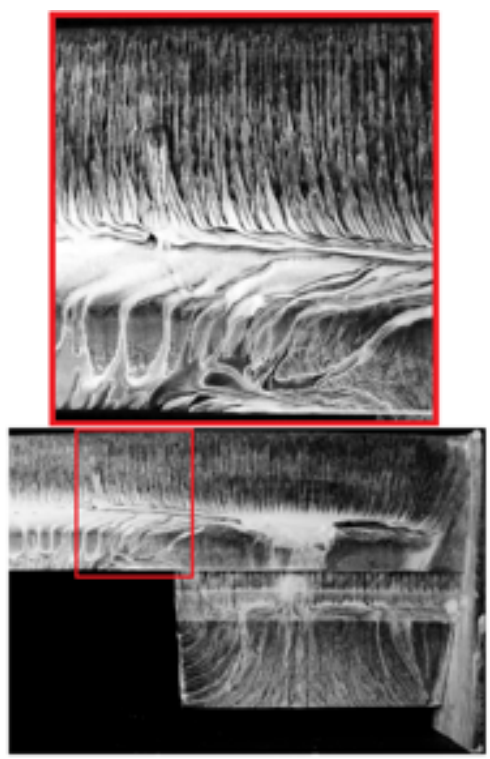

a)

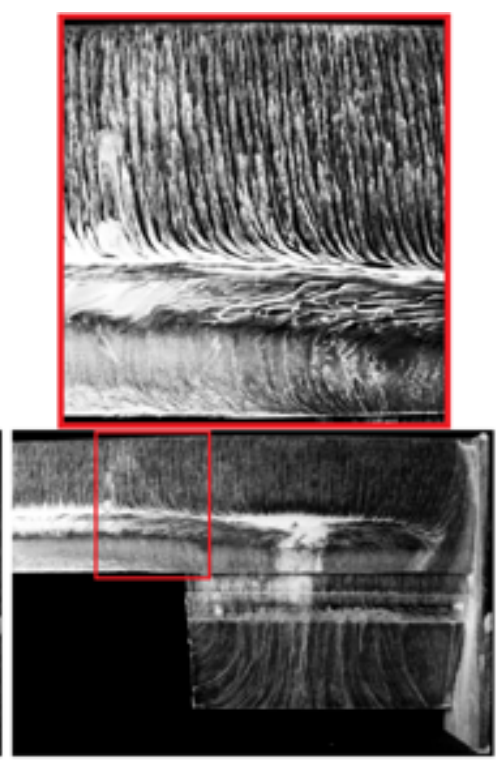

b)

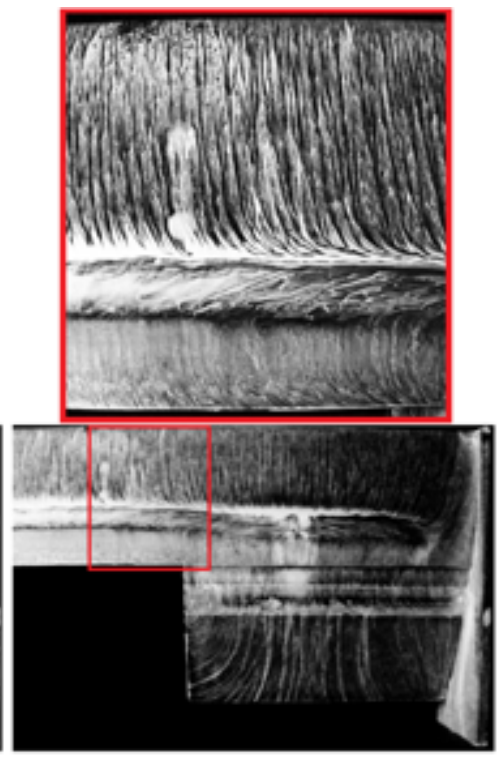

c)

Figure 6 - Suction-surface flow visualisation of multi-element wing at $h / c=0.3125$ for varying Reynolds numbers a) $R$

$\left.=1.63 \times 10^{5}, b\right) \operatorname{Re}=2.04 \times 10^{5}$, and c) $\operatorname{Re}=2.44 \times 10^{5}$ 
Journal of Fluids Engineering

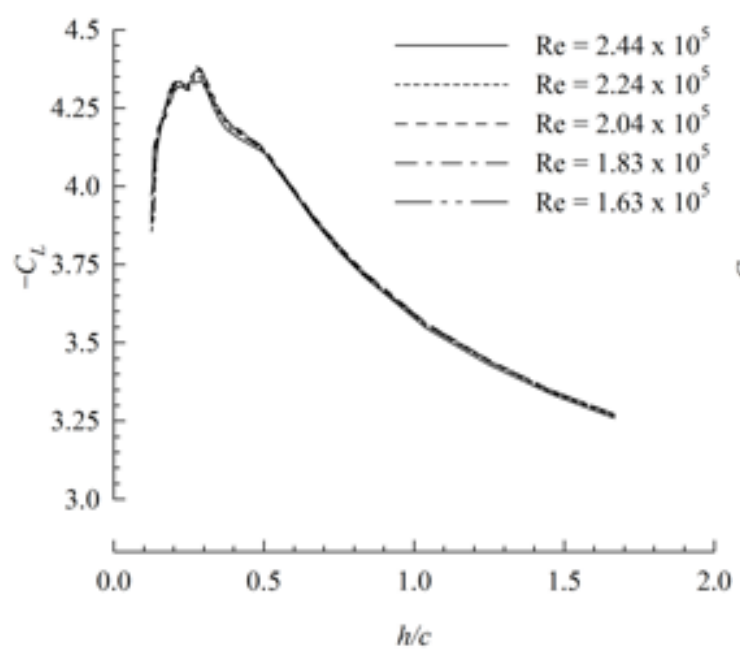

a)

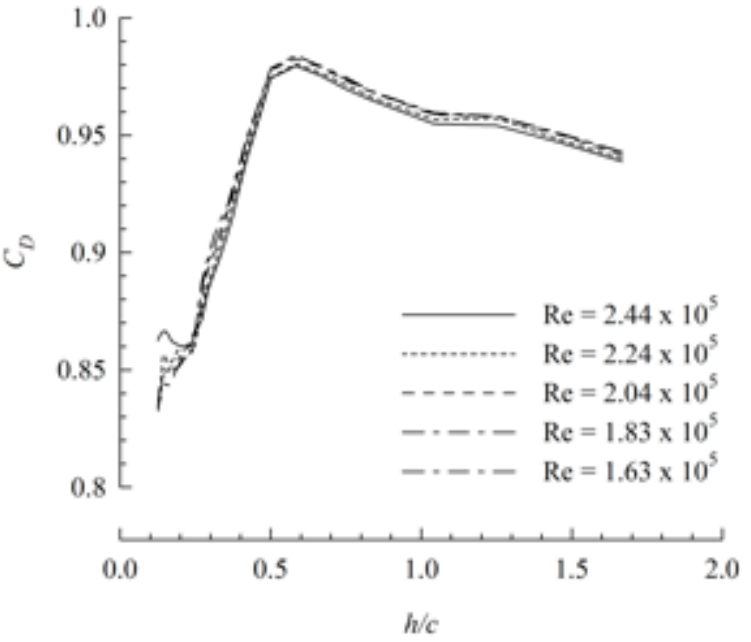

b)

Figure 7 - Formula-One wing: variation with ground clearance $(h / c)$ of a) downforce and b) drag at varying Reynolds number

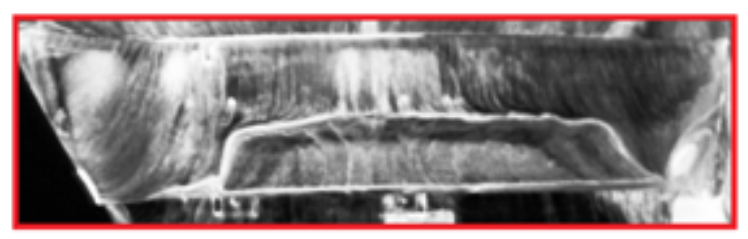

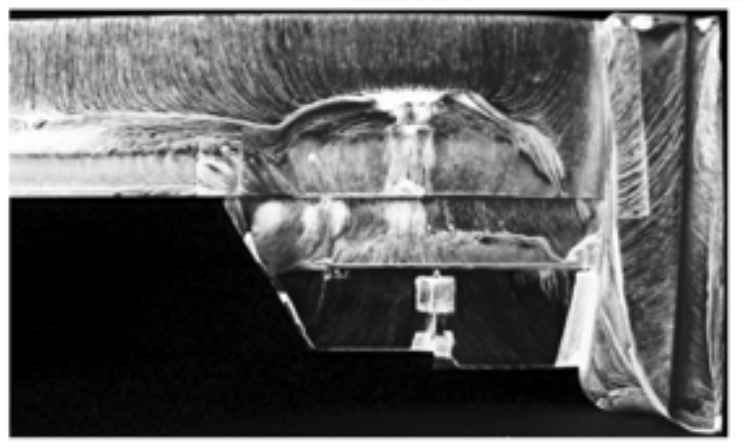

a)

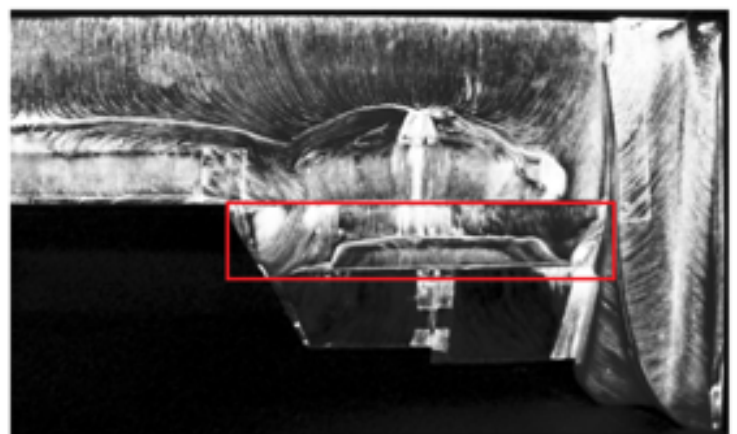

b)

Figure 8 - Suction-surface flow visualisation of Formula-One wing at $h / c=0.3125$ for varying Reynolds numbers a) Re

$=1.63 \times 10^{5}$ and $\left.b\right) \operatorname{Re}=2.44 \times 10^{5}$ 

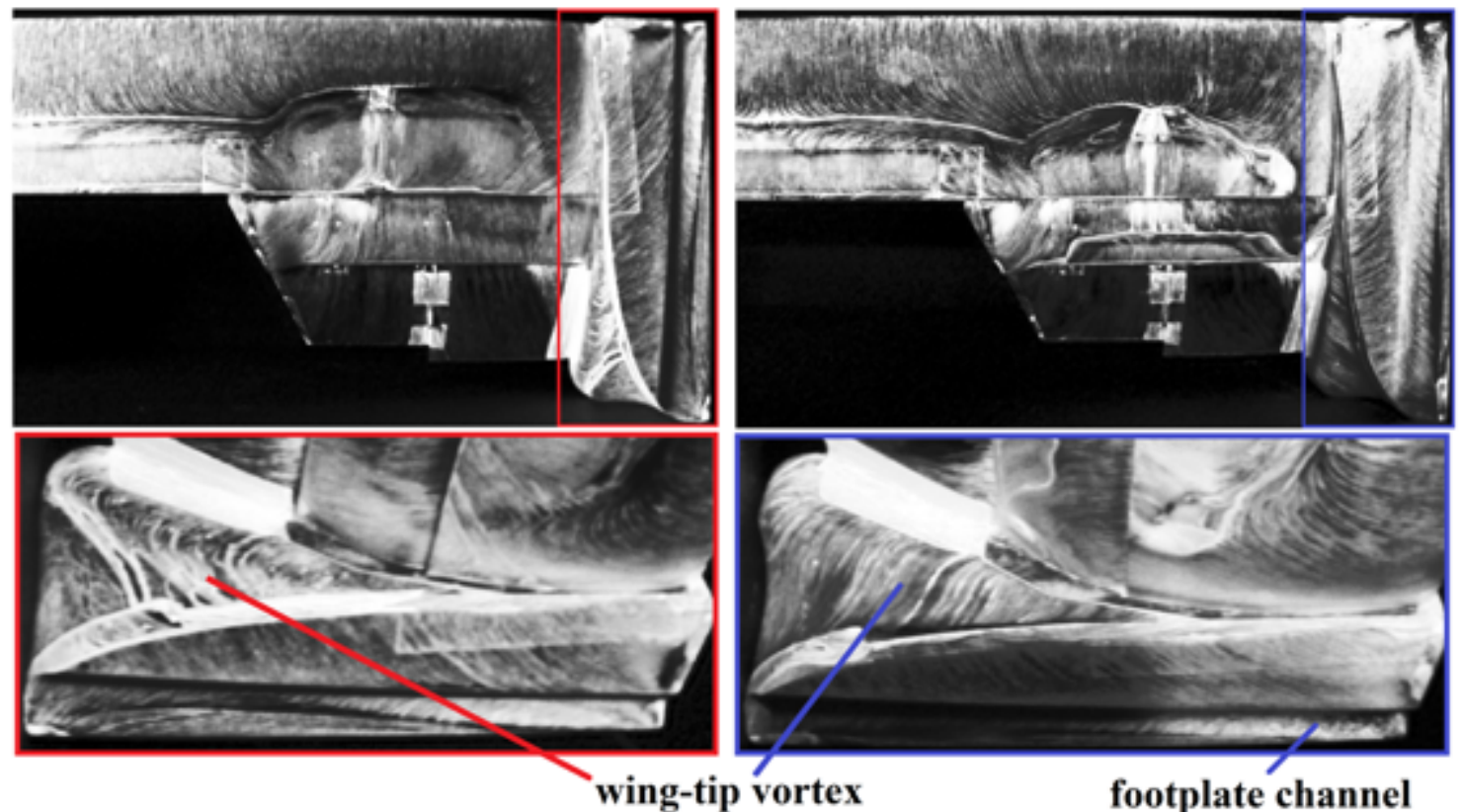

a)

b)

Figure 9 - Flow visualisation of F1 wing suction-surfaces (top) and inner endplate (bottom) at a) $h / c=0.125$ and b) $h / c$

$=0.3125\left(\operatorname{Re}=2.44 \times 10^{5}\right)$

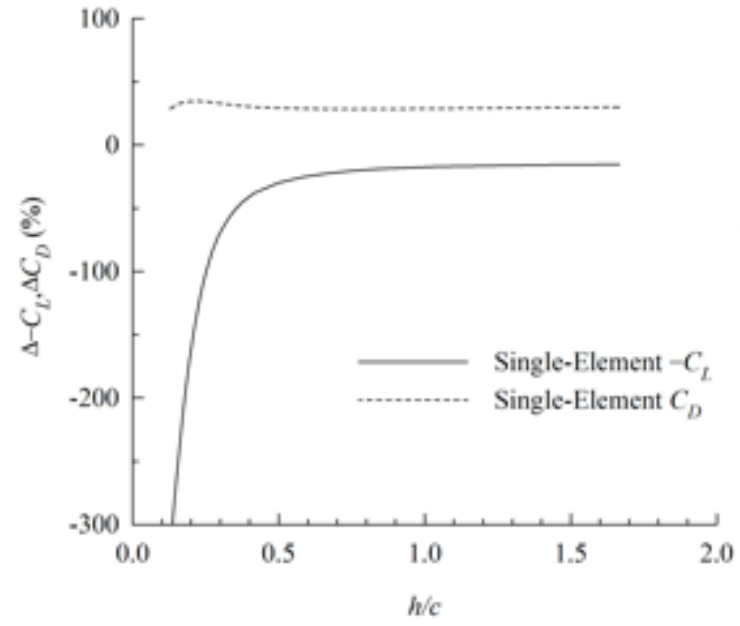

a)

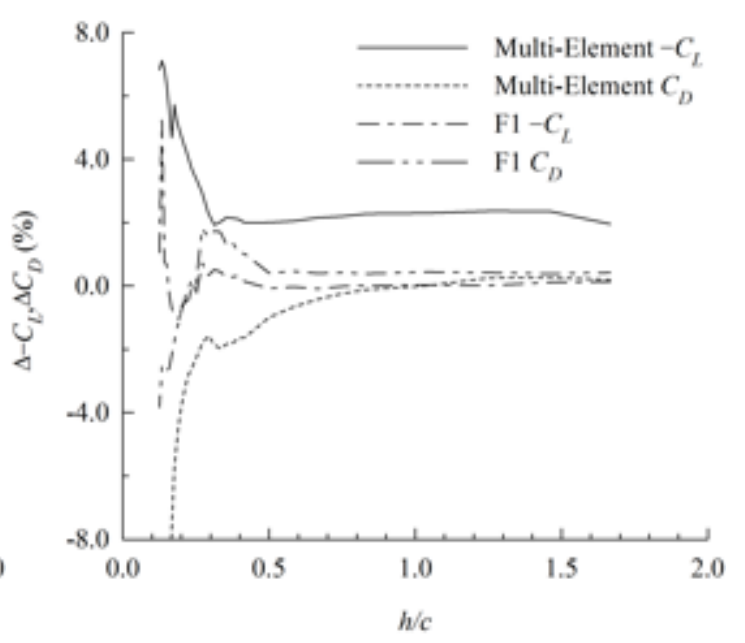

b)

Figure 10 - Reynolds-number dependency of downforce and drag coefficients of a) single-element wing and b) multielement and F1 wing configurations (percentage difference between $\operatorname{Re}=2.44 \times 10^{5}$ and $1.63 \times 10^{5}$ results) 